\section{How cells keep scale}

During early animal embryogenesis rapid cell cycles in the absence of substantial cell growth result in the progressive reduction of cell size. Although it has been observed that nuclear and mitotic spindle size decrease when cell volume is reduced, how intracellular scaling occurs remains elusive. Brownlee and Heald now suggest that the partitioning of importin- $\alpha$ at the plasma membrane, driven by palmitoylation, contributes to intracellular scaling.

Importin- $\alpha$ is a nucleocytoplasmic transport factor that has been shown to associate with the plasma membrane and become less abundant in the cytoplasm of cells in Xenopus laevis embryos during early development. The authors found that this plasma membrane association is driven by importin- $\alpha$ palmitoylation. Moreover, the calculated increase in cell surfaceto-volume ratio associated with early X. laevis development is consistent with a dynamic redistribution of importin- $a$ from the cytoplasm to the membrane with decreasing cell size. This indicates that palmitoylated importin- $\alpha$ can respond to changes in cell size and relocate to the plasma membrane as cell size decreases.

In X. laevis egg extracts, pharmacological blockade of palmitoylation increased spindle length and nuclear size. The opposite effect was observed when de-palmitoylation was inhibited, suggesting that palmitoylation and membrane association of importin- $\alpha$ support nuclear and spindle size reduction. This was confirmed in physiologically relevant conditions - in microfluidic cell-like droplets of varying sizes, in $X$. laevis embryos and in cultured human cells.

Increased palmitoylation was associated with reduced interaction of importin- $\alpha$ with its cargoes (nuclear localization signal (NLS)-containing proteins), including kinesin Kif2a, which depolymerizes spindle microtubules, and the nuclear lamina component lamin B3, which is a limiting factor for nuclear growth. Thus, palmitoylation and membrane localization of importin-a drive nuclear and spindle downscaling by impeding the interaction of importin- $\alpha$ with its cargoes, thereby modulating their cellular distribution and

palmitoylated importin-a can respond to changes in cell size and relocate to the plasma membrane function. How importin- $\alpha$ palmitoylation is regulated and how this modification and membrane partitioning affect other NLS-containing cargoes of importin- $\alpha$ remain to be investigated.

Paulina Strzyz

ORIGINAL ARTICLE Brownlee, C. \& Heald, R. Importin a partitioning to the plasma membrane regulates intracellular scaling. Cell https://doi.org/ 10.1016/j.cell.2018.12.001 (2019)

\section{$\Rightarrow$ CELL MIGRATION}

\section{Forcing through barriers}

Matrix metalloproteinases (MMPs) degrade the basement membrane - a dense extracellular matrix that surrounds all tissues, thereby facilitating cell invasion. Kelley et al. now reveal a compensatory mechanism of cell invasion in the absence of MMPs, relying on increased polymerization of branched actin.

To study cell invasion in vivo, the authors used Caenorhabditis elegans anchor cells, which are specialized uterine cells that invade through the basement membrane into the underlying vulval epithelium during worm development. They generated mutants of five MMPs that localize at the site of anchor cell invasion. Interestingly, even in the absence of all five MMPs (MMP-), anchor cell invasion persisted, although it was delayed by $\sim 1 \mathrm{~h}$; this was associated with the generation of large protrusions — in place of small invadopodia characteristic of wild-type cells - and pronounced physical displacement of basement membrane components away from the invasion site, rather than basement membrane degradation. Thus, anchor cells adapt to the loss of MMPs and efficiently invade the tissue by physically breaching the basement membrane.

Branched actin networks generated by the Arp2/3 complex were previously implicated in anchor cell invasion. Removal of an Arp2/3 subunit by RNAi nearly completely blocked invasion of $\mathrm{MMP}^{-}$cells. In addition, Arp2/3 and $\mathrm{F}$-actin were enriched at the invasive front in $\mathrm{MMP}^{-}$cells compared with wild-type cells. Thus, increased formation of branched actin networks driven by Arp2/3 supports anchor cell invasion in the absence of MMPs.

A genome-wide synthetic RNAi screen further identified the mitochondrial ATP/ADP translocase ANT1.1 as a factor promoting MMP-independent invasion of anchor cells. Accordingly, mitochondria were enriched at the invasive front in $\mathrm{MMP}^{-}$cells, where they were tightly associated with F-actin. ATP production was also increased at the invasive front of MMP- cells, suggesting that enhanced ATP

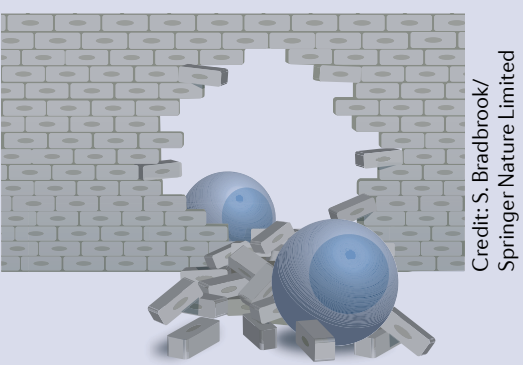

production at the invasive front supports increased branched actin assembly and MMP-independent invasion.

Thus, MMPs are not absolutely required for cell invasion in vivo - in the absence of MMPs cells can physically breach basement membrane barriers using large protrusions enriched in branched actin and ATP-generating mitochondria. These findings may explain the failure of MMP inhibitors as cancer therapeutics and could inform new approaches to block cancer metastasis.

Paulina Strzyz

ORIGINAL ARTICLE Kelley, L. C. et al. Adaptive F-actin polymerization and localized ATP production drive basement membrane invasion in the absence of MMPs. Dev. Cell https://doi.org/ 10.1016/j.devcel.2018.12.018 (2019) 